\title{
Applications of Nijenhuis geometry: non-degenerate singular points of Poisson-Nijenhuis structures
}

\author{
Alexey V. Bolsinov ${ }^{1,2,3}$ - Andrey Yu. Konyaev ${ }^{2}$. Vladimir S. Matveev ${ }^{4}$
}

Received: 14 January 2020 / Accepted: 27 May 2020 / Published online: 6 January 2021

(c) The Author(s) 2021

\section{Abstract}

We study and completely describe pairs of compatible Poisson structures near singular points of the recursion operator satisfying natural non-degeneracy condition.

Keywords Nijenhuis tensor - Singular point - Compatible symplectic structures . Recursion operator · Bihamiltonian systems $\cdot$ Integrable systems

Mathematics Subject Classification 37J35 $\cdot$ 53D20 $\cdot$ 53D22 $\cdot$ 70G45 $\cdot 70 \mathrm{H} 06$

\section{Introduction}

This paper is a follow-up of our previous work [2] that can be considered as an introduction to Nijenhuis Geometry. Nijenhuis geometry studies Nijenhuis operators,

\begin{abstract}
The work of A. Bolsinov and A. Konyaev was supported by the Russian Science Foundation (Project 17-11-01303). Some results were obtained during the visits of A. Bolsinov and A. Konyaev to Jena supported by DAAD (via Ostpartnerschaft programm) and by University of Jena, and during the research in pairs stay of A. Bolsinov, A. Konyaev and V. Matveev at CIRM Trento. V. Matveev thanks DFG for partial support via Projects MA 2565/4 and MA 2565/6.
\end{abstract}

$\triangle$ Alexey V. Bolsinov

A.Bolsinov@lboro.ac.uk

Andrey Yu. Konyaev

maodzund@yandex.ru

Vladimir S. Matveev

vladimir.matveev@uni-jena.de

1 Department of Mathematical Sciences, Loughborough University, LE11 3TU Loughborough, UK

2 Faculty of Mechanics and Mathematics, Moscow State University, 119992 Moscow, Russia

3 Moscow Center for Fundamental and Applied Mathematics, Moscow, Russia

4 Institut für Mathematik, Friedrich Schiller Universität Jena, 07737 Jena, Germany 
i.e., fields of endomorphisms $L=\left(L_{j}^{i}\right)$ of the tangent space with vanishing Nijenhuis torsion: that is, for any vector fields $\xi, \eta$,

$$
N_{L}(\xi, \eta)=L^{2}[\xi, \eta]-L[L \xi, \eta]-L[\xi, L \eta]+[L \xi, L \eta]=0 .
$$

The paper [2] suggests a programme and general strategy for studying such operators (and demonstrates that the programme is realistic by proving a series of non-trivial results). Important part of the strategy is the study of singular points of Nijenhuis operators.

The goal of this paper is to illustrate how methods, ideas, and results of [2] work in describing singular points of more complicated geometric structures involving Nijenhuis operators as one of their ingredients (cf. [2, Sections 6.2 and 6.3]) and also to give a proof for Theorem 6.3 announced in [2].

The geometric structure we consider in this paper came from the theory of integrable systems, where Nijenhuis operators serve as recursion operators for bihamiltonian structures [7,13,16,17], see also [4,5].

Recall that two Poisson structures $P$ and $\widetilde{P}$ are called compatible, if their sum $P+\widetilde{P}$ is also a Poisson structure. If $P$ and $\widetilde{P}$ are non-degenerate and therefore come from symplectic forms $\omega=P^{-1}$ and $\widetilde{\omega}=\widetilde{P}^{-1}$, then the compatibility condition is equivalent to the property that the recursion operator $L$ given by the relation

$$
\widetilde{\omega}(\cdot, \cdot)=\omega(L \cdot, \cdot)
$$

is Nijenhuis. Since $\widetilde{\omega}$ can be recovered from $\omega$ and $L$, we can reformulate the compatibility condition by saying that a symplectic structure $\omega$ and a Nijenhuis operator $L$ are compatible if

(I) the form $\widetilde{\omega}(\cdot, \cdot)=\omega(L \cdot, \cdot)$ is skew-symmetric, i.e., is a differential 2-form, (II) $\mathrm{d} \widetilde{\omega}=0$, i.e., this form is closed.

In the case when $L$ is non-degenerate, a compatible pair $(\omega, L)$ defines a PoissonNijenhuis structure in the sense of $[13,17]$. However, non-degeneracy of $L$ is not very essential as we can replace it with $L+c \cdot \mathrm{Id}, c \in \mathbb{R}$, and here we will think of compatible pairs $(\omega, L)$ as a natural subclass of Poisson-Nijenhuis structures.

It is natural to ask about local simultaneous canonical form for compatible $\omega$ and $L$. In the case when $L$ is algebraically generic at a point $\mathrm{p} \in M^{2 n}$, i.e., its algebraic type (see the next section for details) remains the same in a certain neighbourhood $U(\mathrm{p})$, the answer was obtained in $[7,17,20]$. We briefly recall some of these results.

Notice, first of all, that condition (I) imposes natural algebraic restrictions on the algebraic type of $L$ : in the Jordan decomposition of $L$ all the blocks can be partitioned into pairs of equal blocks (i.e., of the same size and with the same eigenvalue). In particular, the characteristic polynomial of $L$ is a full square and each eigenvalue has even multiplicity.

If $L$ has $n=\frac{1}{2} \operatorname{dim} M$ distinct real eigenvalues, each of multiplicity 2 , and in addition their differentials are linearly independent at a point $p \in M$, then there exists a local symplectic coordinate system $x_{1}, \ldots, x_{n}, p_{1}, \ldots, p_{n}$ in which $L$ is diagonal with $x_{i}$ being its $i$-th eigenvalue [17]: 


$$
\omega=\sum_{i} d p_{i} \wedge d x_{i}, \quad L=\operatorname{diag}\left(x_{1}, x_{2}, \ldots, x_{n}, x_{1}, x_{2}, \ldots, x_{n}\right)
$$

Equivalently, one can say that the pair $(\omega, L)$ is the direct product of two-dimensional blocks $\left(\omega_{i}, L_{i}\right)$ of the form $\omega_{i}=d p_{i} \wedge d x_{i}, L_{i}=x_{i}$. Id. For further use and without pretending that it is new, let us slightly generalise this result (cf. [7,17,20]). Consider four elementary examples of compatible pairs:

Type $1 . L$ has one real non-constant eigenvalue of multiplicity 2 :

$$
\omega=\mathrm{d} p \wedge \mathrm{d} x, \quad L=\lambda(x, p) \cdot \mathrm{Id} .
$$

Type 2. $L$ has one real constant eigenvalue of multiplicity $2 k$ :

$$
\omega=\sum_{j=1}^{k} \mathrm{~d} p_{j} \wedge \mathrm{d} x_{j}, \quad L=\lambda \cdot \mathrm{Id}, \quad \lambda \in \mathbb{R}
$$

Type 3. $L$ has one pair of non-constant complex conjugate eigenvalues of multiplicity 2:

$$
\omega=\operatorname{Re}(\mathrm{d} z \wedge \mathrm{d} w), \quad L=\alpha(z, w) \cdot \operatorname{Id}+\beta(z, w) \cdot J
$$

where $z=x+\mathrm{i} y, w=u+\mathrm{i} v, J$ denotes the corresponding complex structure and $\alpha(z, w)+\mathrm{i} \beta(z, w)$ is a holomorphic function in $z$ and $w$.

Type 4. $L$ has one pair of constant complex conjugate eigenvalues of multiplicity $2 k$ :

$$
\omega=\operatorname{Re}\left(\sum_{j=1}^{k} \mathrm{~d} z_{j} \wedge \mathrm{d} w_{j}\right), \quad L=\alpha \cdot \operatorname{Id}+\beta \cdot J,
$$

where $z_{j}=x_{j}+\mathrm{i} y_{j}, w_{j}=u_{j}+\mathrm{i} v_{j}, J$ denotes the corresponding complex structure and $\alpha+\mathrm{i} \beta \in \mathbb{C}, \beta \neq 0$.

Theorem 1.1 (Folklore) Let $\omega$ and L be compatible. Suppose that L is semisimple and algebraically generic in a neighbourhood of $p \in M$. Then the pair $(\omega, L)$ locally splits into a direct product of 'elementary blocks' of four types described above. If $\mathrm{d} \lambda(p) \neq 0$ or $\mathrm{d}(\alpha(p)+\mathrm{i} \beta(p)) \neq 0$ for some real or complex eigenvalue, then in the corresponding 'elementary block' we may set $\lambda=x$ (see Type 1 ) and $\alpha+\mathrm{i} \beta=z$ (see Type 3).

Notice that to admit a compatible symplectic partner, a semisimple algebraically generic Nijenhuis operator $L$ should satisfy the following additional condition: nonconstant eigenvalues of $L$ must be all of multiplicity 2 .

If $L$ is algebraically generic but not necessarily semisimple, the description (rather non-trivial) of compatible pairs $(\omega, L)$ was obtained by Turiel [20] under some additional assumptions on the differentials of $\operatorname{tr} L^{k}, k=1, \ldots, n$. These assumptions 
basically mean that each eigenvalue is either constant or its differential does not vanish.

The goal of the present paper is to give a simultaneous canonical form for $(\omega, L)$ at typical singular points, where the algebraic type of $L$ changes.

\section{Statement of the main result}

If we want to study singularities of Poisson-Nijenhuis structures (cf. [1, Problem 5.17]), then it is natural to ask which Nijenhuis operators admit compatible symplectic structures and what is a simultaneous canonical form for $\omega$ and $L$ near a (possibly singular) point $\mathrm{p} \in M$ ?

Let us explain in more detail what kind of singularities we have in mind. For a symplectic structure $\omega$, all points $\mathrm{p} \in M$ are obviously equivalent to each other (in other words, there are no singular points). However it is not the case for an operator $L$, since its algebraic properties may essentially vary from point to point. More precisely, at each point $\mathrm{p} \in M$, we can define the algebraic type (or Segre characteristic, see e.g. [6]) of the operator $L(\mathrm{p}): T_{\mathrm{p}} M \rightarrow T_{\mathrm{p}} M$ as the structure of its Jordan canonical form which is characterised by the sizes of Jordan blocks related to each eigenvalue $\lambda_{i}$ of $L(\mathrm{p})$ (the specific values of $\lambda_{i}$ 's are not important here). Following [2, Definition 2.7], we call a point $p \in M$ algebraically generic, if the algebraic type of $L$ does not change in some neighbourhood $U(\mathrm{p}) \subset M$. Otherwise, we say that $\mathrm{p}$ is singular.

Study of singularities of Nijenhuis and other related structures is an important part of the programme suggested in [2] (see also [12]). Actually, in most branches of geometry understanding of singular points of relevant geometric structures plays a central role.

Among singular points, we distinguish (see [2, Definition 2.10]) an important subclass of the so-called differentially non-degenerate singular points $\mathrm{p} \in M$ which are characterised by the property that the differentials of the functions $\operatorname{tr} L^{k}, k=$ $1, \ldots, \operatorname{dim} M$, are linearly independent at $p$. Instead of the traces of powers of $L$ one can equivalently consider the coefficients $\sigma_{1}, \ldots, \sigma_{m}, m=\operatorname{dim} M$, of the characteristic polynomial of $L$ :

$$
\chi_{L}(t)=\operatorname{det}(t \cdot \operatorname{Id}-L)=t^{m}+\sigma_{1} t^{m-1}+\cdots+\sigma_{m-1} t+\sigma_{m} .
$$

Relation (I), however, implies that the eigenvalues of $L$ always have even multiplicity, which in turn implies that $L$ cannot be differentially non-degenerate (since the characteristic polynomial is a full square and has at most $\frac{1}{2} \operatorname{dim} M$ functionally independent coefficients).

As a natural analog of differential non-degeneracy in this setup we suggest the condition that the differentials of the functions $\operatorname{tr} L, \operatorname{tr} L^{2}, \ldots, \operatorname{tr} L^{n}, n=\frac{1}{2} \operatorname{dim} M$, are linearly independent at a given (singular) point $p \in M$. In terms of the characteristic polynomial of $L$ which is a full square (of a polynomial with smooth coefficients) in our case

$$
\chi_{L}(t)=\left(t^{n}+h_{1} t^{n-1}+\cdots+h_{n-1} t+h_{n}\right)^{2}, \quad m=2 n=\operatorname{dim} M .
$$


This condition amounts to linear independence of the differentials $\mathrm{d} h_{1}(\mathrm{p}), \ldots, \mathrm{d} h_{n}(\mathrm{p})$. Our goal is to study local canonical forms for $\omega$ and $L$ at such points. This problem is clearly important for finite dimensional integrable systems and we do hope that it might also be important for understanding of bihamiltonian structures in infinite dimension appearing in the theory of integrable ODEs and PDEs. The main result of this paper is the following theorem whose weaker version is announced in [2].

Theorem 2.1 Let $\omega$ and L be compatible (i.e., define a Poisson-Nijenhuis structure on $\left.M^{2 n}\right)$ and real-analytic. Suppose that at a point $p \in M^{2 n}$, the differentials $\mathrm{d} \operatorname{tr} L, \ldots, \mathrm{d} \operatorname{tr} L^{n}$ are linearly independent. Then there exists a local coordinate system $x_{1}, \ldots, x_{n}, p_{1}, \ldots, p_{n}$ such that $\omega=\sum_{i} \mathrm{~d} x_{i} \wedge \mathrm{d} p_{i}$ and $L$ is given by the matrix

$$
\left(\begin{array}{ll}
A & 0_{n} \\
S & A^{\top}
\end{array}\right)
$$

where

$$
A=\left(\begin{array}{ccccc}
-x_{1} & 1 & 0 & \cdots & 0 \\
-x_{2} & 0 & 1 & \ddots & \vdots \\
\vdots & \vdots & \ddots & \ddots & 0 \\
-x_{n-1} & \vdots & & \ddots & 1 \\
-x_{n} & 0 & \cdots & \cdots & 0
\end{array}\right), \quad S=\left(\begin{array}{ccccc}
0 & -p_{2} & -p_{3} & \cdots & -p_{n} \\
p_{2} & 0 & 0 & \cdots & 0 \\
p_{3} & 0 & \vdots & \ddots & \vdots \\
\vdots & \vdots & \vdots & \ddots & \vdots \\
p_{n} & 0 & 0 & \cdots & 0
\end{array}\right)
$$

$0_{n}$ is the zero $n \times n$-matrix, and $A^{\top}$ denotes the transpose of $A$.

Remark 2.2 We would like to point out that this theorem mainly concerns singular points of Poisson-Nijenhuis structures, i.e., those at which the algebraic type of $L$ changes (recall that the case of algebraically generic points has basically been covered by the results of $[7,17,20])$. The typical and most important example of such a situation is a "perturbation of a Jordan block" when all the eigenvalues of $L$ simultaneously vanish at the point $p \in M^{2 n}$. In this situation, the $x$-coordinates of $p$ are $(0,0, \ldots, 0)$. However, formulas (1) and (2) are universal in the sense that they are applicable to both algebraically generic and singular points of any algebraic kind under the only condition that the differentials $\mathrm{d} \operatorname{tr} L, \ldots, \mathrm{d} \operatorname{tr} L^{n}$ are linearly independent.

More precisely, if a Poisson-Nijenhuis structure $(L, \omega)$ satisfies the conditions of Theorem 2.1 and in addition, at the point $\mathrm{p} \in M^{2 n}$, the operator $L$ has $s$ distinct eigenvalues $\lambda_{1}, \ldots, \lambda_{s}$ (some of which can be complex conjugate) of multiplicities $2 k_{1}, \ldots, 2 k_{s}$, then the local canonical form for $L, \omega$ is given by (1) and (2) where the $x$-coordinates $x_{1}^{0}, \ldots, x_{n}^{0}$ of the point $\mathrm{p}$ are the coefficients of the polynomial

$$
\prod_{i=1}^{s}\left(t-\lambda_{i}\right)^{k_{i}}=t^{n}+x_{1}^{0} t^{n-1}+x_{2}^{0} t^{n-2}+\cdots+x_{n-1}^{0} t+x_{n}^{0},
$$

that is, the square root of the characteristic polynomials $\chi_{L(p)}$.

The rest of the paper is devoted to the proof of Theorem 2.1. First we discuss one "cohomological" property of gl-regular operators serving as a key step in our proof 
and also interesting on its own. This property uses some existence and uniqueness results from general theory of PDEs that we clarify in Appendix A. The proof of Theorem 2.1 is given in Sect. 4. In Appendix B we formulate an alternative version of Theorem 2.1.

All the objects we are dealing with (functions, manifolds, maps) are assumed to be real-analytic.

\section{One cohomology-like property of gl-regular Nijenhuis operators}

Let $V$ be a finite-dimensional vector space. We will say that an operator $A: V \rightarrow V$ is gl-regular if the dimension of its conjugacy class $\mathcal{O}(A)=\left\{P A P^{-1}: P \in \mathrm{GL}(V)\right\}$ is maximal (i.e., equals $n^{2}-n$ ). Equivalently, this regularity condition means that in an appropriate basis the matrix of $A$ takes the so-called companion form as in (7). Typical examples of gl-regular operators include a semisimple operator with distinct eigenvalues and a single Jordan block.

To clarify the relation between the gl-regularity condition and our main Theorem 2.1, it is worth mentioning that the linear independence of the differentials $\mathrm{d} \operatorname{tr} A(\mathrm{p}), \ldots, \mathrm{d} \operatorname{tr} A^{n}(\mathrm{p})$ automatically implies that $A(\mathrm{p})$ is gl-regular. ${ }^{1}$ Notice that $A$ and $L$ from Theorem 2.1 are related by (1), hence $\operatorname{tr} L^{k}=2 \operatorname{tr} A^{k}$ so that the assumptions of this theorem imply gl-regularity of the block $A$. These two operators $A$ and $L$ are related via the Turiel extension construction discussed in Appendix B. However in this section, the reader should think of $A$ just as an operator defined on an $n$-dimensional manifold $Q$ and not related to $L$ in any sense.

Proposition 3.1 Let A be a (real-analytic) Nijenhuis operator which is gl-regular at a point $\mathrm{p} \in Q$. Assume that $\Omega$ is a closed (real-analytic) 2-form such that the form

$$
\Omega_{A}(\xi, \eta)=\Omega(A \xi, \eta)+\Omega(\xi, A \eta)
$$

is also closed. Then locally in some neighbourhood of $\mathrm{p} \in Q$ there exists a (realanalytic) function $U$ such that

$$
\Omega=\mathrm{d}\left(A^{*} \mathrm{~d} U\right) \text {. }
$$

Remark 3.2 Note that the gl-regularity condition is essential. It can be easily checked that the statement of Proposition 3.1 fails for the operator $A=\left(\begin{array}{cc}x_{1} & 0 \\ 0 & x_{2}\end{array}\right)$ at those points $\mathrm{p} \in Q$ where the eigenvalues collide, i.e., $x_{1}=x_{2}$.

Remark 3.3 Proposition 3.1 can be interpreted in terms of the Nijenhuis differential $[13,15,18]$. Denote by $\Lambda^{k}(Q)$ the module of differential $k$-forms on a manifold $Q$, $\Lambda^{0}$ are functions on $Q$ (definition works for both smooth and analytic cases) and $\mathrm{d}: \Lambda^{k} \rightarrow \Lambda^{k+1}$ is the standard differential of $k$-forms.

\footnotetext{
${ }_{1}^{1}$ This is a purely algebraic fact, a particular case of the famous Kostant theorem [14, Theorem 0.1], which can be easily verified in our case related to the Lie algebra $\mathrm{gl}(n)$.
} 
Given an operator $A$, one can define a new differential $\mathrm{d}_{A}: \Lambda^{k} \rightarrow \Lambda^{k+1}$ which is completely determined by the following properties (see [18, Appendix]):

$$
\begin{aligned}
& \text { 1. } \mathrm{d}_{A} f=A^{*} \mathrm{~d} f, \quad f \in \Lambda^{0}, \\
& \text { 2. } \mathrm{d}_{A}(\alpha+\beta)=\mathrm{d}_{A} \alpha+\mathrm{d}_{A} \beta, \quad \alpha, \beta \in \Lambda^{k}, \\
& \text { 3. } \mathrm{d}_{A}(\alpha \wedge \beta)=\mathrm{d}_{A} \alpha \wedge \beta+(-1)^{k} \alpha \wedge \mathrm{d}_{A} \beta, \quad \alpha \in \Lambda^{k}, \quad \beta \in \Lambda^{s}, \\
& \text { 4. } \mathrm{d}_{A} \mathrm{~d}+\mathrm{dd}_{A}=0 .
\end{aligned}
$$

If $\mathcal{N}_{A}=0$, we have that $\mathrm{d}_{A}^{2}=0$ (see [13, Corollary 6.1] for $E=T Q, N=A$ and $\mu$ being standard commutator of vector fields).

The explicit formula for the operator $\mathrm{d}_{A}: \Lambda^{k} \rightarrow \Lambda^{k+1}$ is as follows [13]:

$$
\mathrm{d}_{A}=\left[i_{A}, \mathrm{~d}\right]
$$

where $i_{A}: \Lambda^{m} \rightarrow \Lambda^{m}$ (for any $m \in \mathbb{N}$ ) is defined by

$$
\begin{aligned}
\left(i_{A} \alpha\right)\left(\xi_{1}, \xi_{2}, \ldots, \xi_{m}\right)=\alpha\left(A \xi_{1}, \xi_{2}, \ldots, \xi_{m}\right) & +\alpha\left(\xi_{1}, A \xi_{2}, \ldots, \xi_{m}\right) \\
& +\cdots+\alpha\left(\xi_{1}, \xi_{2}, \ldots, A \xi_{m}\right)
\end{aligned}
$$

so that for 2-forms $i_{A}$ is essentially given by (3), i.e., $i_{A} \Omega=\Omega_{A}$. We assume that $i_{A}(f)=0$ for any function $f$.

Thus, Proposition 3.1 can be reformulated as follows: given a 2 -form $\Omega$ such that $\mathrm{d} \Omega=0$ and $\mathrm{d}_{A} \Omega=0$ (the latter formula follows from (3) combined with (5)), one can always find a function $U$ such that $\Omega=\mathrm{dd}_{A} U$. In this view, Proposition 3.1 reminds the standand Poincaré lemma for de Rham cohomology and is an important property of the bidifferential complex related to $\mathrm{d}_{\text {and }} \mathrm{d}_{A}$ in the case when $A$ is gl-regular. This explains the title of this section.

The goal of the rest of this section is to prove Proposition 3.1. Recall that we are mainly interested in those points $\mathrm{p} \in Q$ where $A$ changes its algebraic type. The key example of such a situation is a perturbation of a Jordan block, i.e., $A(p)$ is similar to a single Jordan block with zero eigenvalue, but generically the operator $A(q), q \in V(p)$, is semisimple with distinct eigenvalues (as in (2)).

We however first consider the case when $A(p)$ is regular and semisimple.

Lemma 3.4 In the assumptions of Proposition 3.1, let A(p) be semisimple with distinct eigenvalues. Then the statement of Proposition 3.1 holds. Moreover, the general solution $U$ of (4) depends on (is parametrised by) $n$ arbitrary functions of one variable.

Proof Consider a local coordinate system $y_{1}, \ldots, y_{n}$ in which $A$ takes diagonal form

$$
A=\operatorname{diag}\left(\lambda_{1}\left(y_{1}\right), \ldots, \lambda_{n}\left(y_{n}\right)\right) .
$$

Since $A$ is Nijenhuis, such a coordinate system exists $[9,19]$.

Consider a closed 2-form $\Omega=\sum_{i<j} \omega_{i j}(y) \mathrm{d} y_{i} \wedge \mathrm{d} y_{j}$ satisfying (3), i.e., $\Omega_{A}=$ $\sum_{i<j}\left(\lambda_{i}+\lambda_{j}\right) \omega_{i j}(y) \mathrm{d} y_{i} \wedge \mathrm{d} y_{j}$ is also closed. 
We need to solve (4) which takes, in coordinates $y_{1}, \ldots, y_{n}$, the following form:

$$
\left(\lambda_{j}-\lambda_{i}\right) \frac{\partial^{2} U}{\partial y_{i} y_{j}}=\omega_{i j}
$$

or, equivalently,

$$
\frac{\partial^{2} U}{\partial y_{i} \partial y_{j}}=\frac{\omega_{i j}}{\lambda_{j}-\lambda_{i}}, \quad i \neq j
$$

This equation is consistent if and only if

$$
\frac{\partial}{\partial y_{k}}\left(\frac{\omega_{i j}}{\lambda_{i}-\lambda_{j}}\right)=\frac{\partial}{\partial y_{i}}\left(\frac{\omega_{j k}}{\lambda_{j}-\lambda_{k}}\right)=\frac{\partial}{\partial y_{j}}\left(\frac{\omega_{k i}}{\lambda_{k}-\lambda_{i}}\right)
$$

for $i \neq j \neq k \neq i$, which can also be rewritten as

$$
\frac{\partial \omega_{i j}}{\partial y_{k}}\left(\lambda_{i}-\lambda_{j}\right)^{-1}=\frac{\partial \omega_{j k}}{\partial y_{i}}\left(\lambda_{j}-\lambda_{k}\right)^{-1}=\frac{\partial \omega_{k i}}{\partial y_{j}}\left(\lambda_{k}-\lambda_{i}\right)^{-1} .
$$

These two linear relations follow from (and in fact, are equivalent to)

$$
\frac{\partial \omega_{i j}}{\partial y_{k}}+\frac{\partial \omega_{j k}}{\partial y_{i}}+\frac{\partial \omega_{k i}}{\partial y_{j}}=0 \quad(\text { closedness of } \Omega)
$$

and

$$
\frac{\partial \omega_{i j}}{\partial y_{k}}\left(\lambda_{i}+\lambda_{j}\right)+\frac{\partial \omega_{j k}}{\partial y_{i}}\left(\lambda_{j}+\lambda_{k}\right)+\frac{\partial \omega_{k i}}{\partial y_{j}}\left(\lambda_{k}+\lambda_{i}\right)=0 \quad\left(\text { closedness of } \Omega_{A}\right) .
$$

Hence, (6) (or equivalently (4)) admits a solution $U_{0}$. Since (6) is linear in $U$, its general solution is defined modulo solutions of the corresponding homogeneous system

$$
\frac{\partial^{2} U}{\partial y_{i} \partial y_{j}}=0
$$

that is,

$$
U=U_{0}\left(y_{1}, \ldots, y_{n}\right)+u_{1}\left(y_{1}\right)+\cdots+u_{n}\left(y_{n}\right) .
$$

In other words, $U$ depends on $n$ functions in one variable, as stated.

Remark 3.5 One can similarly check that Proposition 3.1 holds under the additional condition that $\mathrm{p} \in Q$ is algebraically generic, i.e., the algebraic type of $A$ remains locally constant in a neighbourhood of $\mathrm{p} \in Q$. The splitting theorem [2, Section 3.2] allows one to assume without loss of generality that $A$ has either a single real 
eigenvalue or single pair of complex conjugate eigenvalues. In this case, due to glregularity, $A$ will be similar to a single Jordan block (real or complex) and the general solution of (4) can be found explicitly by using the canonical forms for such operators from [2]. We omit details of this computation here, although the explicit description of functions $U$ satisfying the condition $\mathrm{d}\left(A^{*} \mathrm{~d} U\right)=0$ for $A$ being a Nijenhuis Jordan block, could be interesting for several reasons. We will provide such a description in another paper.

The following statement is a simple fact from Linear Algebra. It is well known that every gl-regular operator $A$ can be reduced (at one single point $\mathrm{p} \in Q$ ) to a companion form, ${ }^{2}$ i.e.,

$$
A(\mathrm{p})=A_{\text {comp }}=\left(\begin{array}{cccc}
-\sigma_{1} & 1 & & \\
\vdots & 0 & \ddots & \\
-\sigma_{n-1} & & \ddots & 1 \\
-\sigma_{n} & 0 & \ldots & 0
\end{array}\right),
$$

where $\sigma_{i}$ will be automatically the coefficients of the characteristic polynomial $\chi_{A}(t)=\operatorname{det}(t \cdot \operatorname{Id}-A)$.

Lemma 3.6 Let $x_{1}, \ldots, x_{n}$ be a local coordinate system in a neighbourhood of $p \in Q$

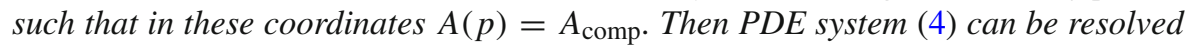
w.r.t. all second order derivatives of $U$ except those of the form $U_{x_{i} x_{n}}, i=1, \ldots, n$, i.e., can be rewritten in the form

$$
U_{x_{i} x_{j}}:=\frac{\partial^{2} U}{\partial x_{i} \partial x_{j}}=h_{i j}\left(x_{1}, \ldots, x_{n}, U_{x_{1}}, \ldots, U_{x_{n}}, U_{x_{1} x_{n}}, \ldots, U_{x_{n} x_{n}}\right)
$$

$i, j=1, \ldots, n-1$.

Proof At the point $p \in Q$ itself, the proof is a simple exercise in Linear Algebra. In all neighbouring points, representation (8) for system (4) holds by continuity.

Remark 3.7 Notice that the possibility to resolve (4) with respect to $\left(n^{2}-n\right) / 2$ (out of $\left.\left(n^{2}+n\right) / 2\right)$ second partial derivatives of $U$, as in Lemmas 3.4 and 3.6, is equivalent to the gl-regularity of $A$.

In order to prove Proposition 3.1 we will show that PDE system (8) possesses sufficiently many solutions (namely, the space of solutions is parametrised by $n$ functions in one variable). To this end, we use the following key property of PDE systems (the proof can be found in Appendix A).

Lemma 3.8 The following properties of PDE system (8) are equivalent:

\footnotetext{
2 In fact, for gl-regular Nijenhuis operators $A$, this can be done simultaneously for all points from some neighbourhood of $p$ by choosing an appropriate local coordinate system [3].
} 
(i) For any initial straight line $x_{1}=a_{1}, \ldots, x_{n-1}=a_{n-1}, a_{i} \in \mathbb{R}$, and any initial conditions given on it

$$
\begin{aligned}
U\left(a_{1}, \ldots, a_{n-1}, x_{n}\right) & =v\left(x_{n}\right), \\
U_{x_{1}}\left(a_{1}, \ldots, a_{n-1}, x_{n}\right) & =v_{1}\left(x_{n}\right), \\
\cdots & \\
U_{x_{n-1}}\left(a_{1}, \ldots, a_{n-1}, x_{n}\right) & =v_{n-1}\left(x_{n}\right),
\end{aligned}
$$

there locally exists a unique real-analytic solution $U\left(x_{1}, \ldots, x_{n}\right)$ of (8).

(ii) The following compatibility conditions identically hold in virtue of (8):

$$
D_{x_{k}} h_{i j}=D_{x_{i}} h_{j k}=D_{x_{j}} h_{k i}
$$

In our case, the functions $h_{i j}$ are linear (but not homogeneous) in the derivatives $U_{x_{i}}$ and $U_{x_{i} x_{n}}$, i.e.,

$$
h_{i j}=\sum_{s=1}^{n} A_{i j}^{s}(x) U_{x_{s} x_{n}}+\sum_{s=1}^{n} B_{i j}^{s}(x) U_{x_{s}}+C_{i j}(x) .
$$

For a given operator $A$ and 2-form $\Omega$, the compatibility conditions (9) take the form

$$
c_{\alpha}(x) \equiv 0, \quad \alpha \in I
$$

where $c_{\alpha}$ are some functions in coordinates $\left(x_{1}, \ldots, x_{n}\right)$ that can be explicitly written in terms of the components of the operator $A, 2$-form $\Omega$, and their partial derivatives, i.e.,

$$
c_{\alpha}(x)=\widetilde{c}_{\alpha}\left(A_{i}^{j}, \frac{\partial A_{i}^{j}}{\partial x_{k}}, \omega_{i j}, \frac{\partial \omega_{i j}}{\partial x_{k}}\right) .
$$

Recall that both $A$ and $\Omega$ are rather special and satisfy very strong conditions. However, straightforward verification of compatibility conditions (10) (and even explicit description of them in terms of $A, \Omega$ and their derivatives) is not an easy task. Instead, we will use the fact that $c_{\alpha}(x)$ is real-analytic in $x$ and for this reason it is sufficient to verify these conditions only for some open subset $V_{0} \subset V(\mathrm{p})$. As such a subset we consider a small neighbourhood $U(\mathrm{q}) \subset V(\mathrm{p})$ of a point $q \in V(p)$ at which $A(\mathrm{q})$ is semisimple (similar verification can be done for $q$ being algebraically generic, ${ }^{3}$ cf. Remark 3.5). According to Lemma 3.4, the general solution $U$ to PDE system (4) (which is equivalent to (8)) depends on $n$ arbitrary real-analytic functions of one variable. Basically, this property is equivalent to condition (i) from Lemma 3.8 and therefore the compatibility conditions (9) from (ii) hold.

However, the collections of $n$ functions parametrising the space of solutions of (4) in Lemmas 3.4 and 3.8 are formally different. So we need to justify the fact that

\footnotetext{
3 Here we use the fact that algebraically generic points form an open and everywhere dense subset.
} 
by choosing appropriate functions $u_{1}\left(y_{1}\right), \ldots, u_{n}\left(y_{n}\right)$ (see Lemma 3.4) we can fulfil arbitrary initial conditions $v\left(x_{n}\right), v_{1}\left(x_{n}\right), \ldots, v_{n-1}\left(x_{n}\right)$ from Lemma 3.8.

Consider the parametrised straight line $\gamma$ given in coordinates $x_{1}, \ldots, x_{n}$ (from Lemma 3.8) as

$$
\gamma(t): \quad x_{1}=a_{1}, \quad x_{2}=a_{2}, \ldots, \quad x_{n-1}=a_{n-1}, \quad x_{n}=t .
$$

The same line in coordinates $y_{1}, \ldots, y_{n}$ (from Lemma 3.4) will be given as

$$
\gamma(t): \quad y_{1}=\phi_{1}(t), \quad y_{2}=\phi_{2}(t), \ldots, \quad y_{n-1}=\phi_{n-1}(t), \quad y_{n}=\phi_{n}(t) .
$$

It can be easily seen that each of these functions $\phi_{i}$ is locally invertible, i.e., $\frac{d \phi_{i}}{d t} \neq 0$ at $t=0$. Indeed, this property means that the entries of the last column $\xi$ of the Jacobi matrix $J=\left(\frac{\partial y}{\partial x}\right)$ (at the origin) are all different from zero. This matrix satisfies the relation $A_{\text {comp }}(x)=J^{-1} A_{\text {diag }}(y) J$ or, equivalently, $J A_{\text {comp }}=A_{\text {diag }} J$. The latter can be interpreted as follows: the columns of $J$ are (in the reverse order) of the form

$$
\xi, A_{\text {diag }} \xi, A_{\text {diag }}^{2} \xi, \ldots, A_{\text {diag }}^{n-1} \xi
$$

In particular, these vectors are linearly independent. For a diagonal matrix $A_{\text {diag }}$ this condition holds if and only if all the components of $\xi \in \mathbb{R}^{n}$ are different from zero, as stated.

The initial conditions (i) from Lemma 3.8 can be rewritten in the form

$$
U(\gamma(t))=v(t), \quad U_{x_{1}}(\gamma(t))=v_{1}(t), \ldots, \quad U_{x_{n-1}}(\gamma(t))=v_{n-1}(t)
$$

It is more convenient to rewrite the first condition in the form $U_{x_{n}}(\gamma(t))=v_{n}(t)=$ $v^{\prime}(t), U(\gamma(0))=c$ (where $c \in \mathbb{R}$ is an arbitrary constant). In other words, we replace $v(t)$ with its derivative $v^{\prime}(t)$ and the value $c \in \mathbb{R}$ at $t=0$.

Using the standard relations $U_{x_{j}}=\sum_{\alpha} \frac{\partial y_{\alpha}}{\partial x_{j}} U_{y_{\alpha}}$ we can rewrite these conditions in coordinates $y_{1}, \ldots, y_{n}$ as follows:

$$
\left.\sum_{\alpha} \frac{\partial y_{\alpha}}{\partial x_{j}} U_{y_{\alpha}}\right|_{\gamma(t)}=v_{j}(t)
$$

and using $U\left(y_{1}, \ldots, y_{n}\right)=U_{0}\left(y_{1}, \ldots, y_{n}\right)+u_{1}\left(y_{1}\right)+\cdots+u_{n}\left(y_{n}\right)$ as

$$
\left.\sum_{\alpha} \frac{\partial y_{\alpha}}{\partial x_{j}}\left(\left(U_{0}\right)_{y_{\alpha}}+u_{\alpha}^{\prime}\right)\right|_{\gamma(t)}=v_{j}(t)
$$

or

$$
u_{\alpha}^{\prime}\left(\phi_{\alpha}(t)\right)=b_{\alpha}(t)+\sum_{\beta} a_{\alpha}^{j}(t) v_{j}(t),
$$


where $b_{\alpha}(t)=-\left(U_{0}\right)_{y_{\alpha}}(\gamma(t)), a_{\alpha}^{j}(t)=\frac{\partial x_{j}}{\partial y_{\alpha}}(\gamma(t))$. Taking into account the fact that $\phi_{\alpha}(t)$ is locally invertible, we see that the derivatives of the functions $u_{1}, \ldots, u_{n}$ can be uniquely reconstructed from $v_{1}, \ldots, v_{n}$ and vice versa as required.

We have shown that in a neighbourhood of any point $\mathrm{q}$ at which $A(\mathrm{q})$ is diagonalisable over $\mathbb{R}$, we can find a solution of (4) with arbitrary initial conditions (i) from Lemma 3.8. This guarantees the fulfilment of the stong compatibility conditions (9) from (ii) in this neighbourhood and therefore (due to real-analyticity) in the whole neighbourhood $V(\mathrm{p})$. The latter, in turn, guarantees the existence of local solutions of (4) in a neighbourhood of $\mathrm{p} \in Q$, completing the proof of Proposition 3.1.

\section{Proof of the main theorem}

Recall that we consider $\omega$ and $L$ to be compatible and real-analytic. We also assume that at a point $\mathrm{p} \in M^{2 n}$ all the eigenvalues of $L$ vanish, but the differentials $\mathrm{d} \operatorname{tr} L, \ldots, \mathrm{d} \operatorname{tr} L^{n}$ are linearly independent.

Since the bilinear form $\widetilde{\omega}(\cdot, \cdot)=\omega(L \cdot, \cdot)$ is skew-symmetric, the characteristic polynomial of $L$ is a full square:

$$
\chi_{L}(t)=\left(t^{n}+h_{1} t^{n-1}+\cdots+h_{n}\right)^{2} .
$$

i.e., the square of a certain polynomial of degree $n$. The condition that the differentials of the functions $\operatorname{tr} L, \ldots, \operatorname{tr} L^{n}$ are linearly independent implies that the differentials of the functions $h_{1}, \ldots, h_{n}$ are linearly independent too and we can take them as the first $n$ coordinates $x_{1}, \ldots, x_{n}$ of a local coordinate system. It is well known that these functions commute with respect to the Poisson bracket related to $\omega$ (see $[7,17]$ ) which, by the Darboux theorem, implies local existence of functions $p_{1}, \ldots, p_{n}$ such that $\left(x_{1}, \ldots, x_{n}, p_{1}, \ldots, p_{n}\right)$ is a canonical coordinate system for $\omega$.

In these coordinates, the operator $L$ takes an "almost required" form. To see this we use the following formula proved in [2]. Let $\sigma_{1}, \ldots, \sigma_{m}, m=\operatorname{dim} M$, be the coefficients of the characteristic polynomial of $L$ :

$$
\chi_{L}(t)=t^{m}+\sigma_{1} t^{m-1}+\sigma_{2} t^{m-2}+\cdots+\sigma_{m-1} t+\sigma_{m}
$$

considered as smooth functions on $M$. Then in any coordinate system $y_{1}, \ldots, y_{m}$, the matrix $L(y)$ satisfies the following relation:

$$
J L(y)=S J, \quad \text { where } S=\left(\begin{array}{cccc}
-\sigma_{1} & 1 & \\
\vdots & 0 & \ddots & \\
-\sigma_{m-1} & \vdots & \ddots & 1 \\
-\sigma_{m} & 0 & \ldots & 0
\end{array}\right)
$$

and $J=\left(\frac{\partial \sigma_{i}}{\partial y_{j}}\right)$ is the Jacobi matrix of the functions $\sigma_{1}, \ldots, \sigma_{m}$ w.r.t. $y_{1}, \ldots, y_{m}$. In our case, the characteristic polynomial $\chi_{L}(t)$ is a full square as in (11) so that its coefficients 
$\sigma_{i}, i=1, \ldots, 2 n$, are some explicit polynomials in $h_{\alpha}=x_{\alpha}, \alpha=1, \ldots, n$, and do not depend on the other half of coordinates $p_{\alpha}, \alpha=1, \ldots, n$. It is now straightforward to check that in coordinates $(x, p)$ the first $n$ rows of the operator $L$ are as in (1), (2). Furthermore, the algebraic compatibility condition (I) implies that the lower right $n \times n$-block of $L$ is transposed to the upper left $n \times n$-block, so that $L$ takes the form

$$
L=\left(\begin{array}{cc}
A & 0 \\
\widehat{S} & A^{\top}
\end{array}\right)
$$

with $A$ as in (2) and $\widehat{S}$ being just a skew-symmetric matrix (again due to condition (I)) whose components may a priori depend on all variables.

Next we use the differential compatibility condition (II) saying that the 2-form $\widetilde{\omega}(\cdot, \cdot)=\omega(L \cdot, \cdot)$ is closed. This form is given by

$$
\widetilde{\omega}=\sum_{i=1}^{n} x_{i} \mathrm{~d} x_{1} \wedge \mathrm{d} p_{i}+\sum_{i, j=1}^{n} \widehat{S}_{i j} \mathrm{~d} x_{i} \wedge \mathrm{d} x_{j}
$$

and its differential is

$$
\begin{aligned}
\mathrm{d} \widetilde{\omega}=-\sum_{i=2}^{n} \mathrm{~d} x_{1} \wedge \mathrm{d} x_{i} \wedge \mathrm{d} p_{i} & +\sum_{i, j, k=1}^{n} \frac{\partial \widehat{S}_{i j}}{\partial x_{k}} \mathrm{~d} x_{k} \wedge \mathrm{d} x_{i} \wedge \mathrm{d} x_{j} \\
& +\sum_{i, j, k=1}^{n} \frac{\partial \widehat{S}_{i j}}{\partial p_{k}} \mathrm{~d} p_{k} \wedge \mathrm{d} x_{i} \wedge \mathrm{d} x_{j}
\end{aligned}
$$

Substituting $\widehat{S}=S+T$ in the formula above, where $S$ is as in (2), we observe that the first sum in (13) cancels with the terms coming from $S$ and we obtain

$$
\mathrm{d} \widetilde{\omega}=\sum_{i, j, k=1}^{n} \frac{\partial T_{i j}}{\partial p_{k}} \mathrm{~d} p_{k} \wedge \mathrm{d} x_{i} \wedge \mathrm{d} x_{j}+\sum_{i, j, k=1}^{n} \frac{\partial T_{i j}}{\partial x_{k}} \mathrm{~d} x_{k} \wedge \mathrm{d} x_{i} \wedge \mathrm{d} x_{j}
$$

Since $\widetilde{\omega}$ is closed, we see that $T_{i j}$ does not depend on the $p$-variables, so that the form $\sum_{i, j=1}^{n} T_{i j}(x) d x_{i} \wedge d x_{j}$ can be viewed as a closed 2 -form $T$ on the local $n$-dimensional coordinate chart $\left(x_{1}, \ldots, x_{n}\right)$.

To complete the proof, we will show that the form $T$ can be "killed" by a suitable canonical transformation of the form

$$
\left(X_{1}, \ldots, X_{n}, P_{1}, \ldots, P_{n}\right)=\left(x_{1}, \ldots, x_{n}, p_{1}+\frac{\partial U}{\partial x_{1}}, \ldots, p_{n}+\frac{\partial U}{\partial x_{n}}\right),
$$

where $U$ is a function of $x_{1}, \ldots, x_{n}$ (called sometimes generating function). This transformation preserves the (canonical) symplectic structure $\omega$ so that we only need to look after the change of $L$. 
The Jacobi matrix of coordinate transformation (14) is $J=\left(\begin{array}{cc}\text { id } & 0 \\ d^{2} U & \text { id }\end{array}\right)$, where id denotes the identity $n \times n$ matrix and $\mathrm{d}^{2} U=\left(\frac{\partial^{2} U}{\partial x_{i} \partial x_{j}}\right)$ is the Hessian matrix of $U$. Hence, after transformation (14) the matrix of $L$ given by (12) takes the form

$$
L_{\text {new }}=J L J^{-1}=\left(\begin{array}{cc}
\mathrm{id} & 0 \\
\mathrm{~d}^{2} U & \mathrm{id}
\end{array}\right)\left(\begin{array}{cc}
A & 0 \\
\widehat{S} & A^{\top}
\end{array}\right)\left(\begin{array}{cc}
\mathrm{id} & 0 \\
-\mathrm{d}^{2} U & \mathrm{id}
\end{array}\right)=\left(\begin{array}{cc}
A & 0 \\
\widetilde{S} & A^{\top}
\end{array}\right) \text {, }
$$

where $\widetilde{S}=\widehat{S}+\mathrm{d}^{2} U \cdot A-A^{\top} \cdot \mathrm{d}^{2} U$. Taking into account the transformation of the $p$-coordinates in $S$, we see that $L_{\text {new }}$ takes the required form (1), (2) if and only if the generating function $U$ satisfies the following equation:

$$
0=T+\mathrm{d}^{2} U \cdot A-A^{\top} \cdot \mathrm{d}^{2} U+\mathrm{d} U \wedge \mathrm{d} x_{1},
$$

which can be equivalently rewritten in a more invariant form

$$
\mathrm{d}\left(A^{*} \mathrm{~d} U\right)=T
$$

that coincides with PDE system (4) treated in the previous section. In order to check the existence of a generating function $U$ solving (15), it remains to verify two conditions from Proposition 3.1 imposed on the 2-form $T$.

They both follow from the fact that $L=\left(\begin{array}{cc}A & 0 \\ \widehat{S} & A^{\top}\end{array}\right)$ with $\widehat{S}=S+T$ and $A$ and $S$ as in (2), is a Nijenhuis operator. It is easily checked by a straightforward computation that vanishing Nijenhuis torsion of $L$ is exactly equivalent to the two geometric conditions we need, namely that the 2 -forms

$$
T=\sum_{i, j=1}^{n} T_{i j} \mathrm{~d} x_{i} \wedge \mathrm{d} x_{j} \quad \text { and } \quad T_{A}=\sum_{i, j, k=1}^{n}\left(A^{k}{ }_{i} T_{k j}+A^{k}{ }_{j} T_{i k}\right) \mathrm{d} x_{i} \wedge \mathrm{d} x_{j}
$$

are closed. This allows us to apply Proposition 3.1 that now guarantees the existence of $U$ solving (15) and hence completes the proof of Theorem 2.1.

\section{Appendix A: Proof of Lemma 3.8}

The goal in this appendix is to prove Lemma 3.8 concerning the solvability of the PDE system

$$
U_{x_{i} x_{j}}=h_{i j}\left(x_{1}, \ldots, x_{n}, U_{x_{1}}, \ldots, U_{x_{n}}, U_{x_{1} x_{n}}, \ldots, U_{x_{n} x_{n}}\right), \quad i, j=1, \ldots, n-1 .
$$

Lemma 3.8 is not a new fact and can be understood as a special case of the CartanKähler theorem (see e.g. [8]). We suppose that people working in the Cartan-Kähler theory would find it trivial. However, deriving Lemma 3.8 from a very general and non-trivial Cartan-Kähler theorem seems to require more work than proving it directly. 
Unfortunately, we have not found this lemma in the required form in the literature, so we will prove it here in order to make the paper self-contained.

As one usually does in the theory of differential equations, we set $f_{1}=U_{x_{1}}, f_{2}=$ $U_{x_{2}}, \ldots, f_{n}=U_{x_{n}}$ and rewrite the above system in the form

$$
F_{x_{i}}=H_{i}\left(x, F, F_{x_{n}}\right), \text { with } F=\left(\begin{array}{c}
f_{1} \\
\vdots \\
f_{k}
\end{array}\right), \quad i=1, \ldots, n-1 .
$$

In our case the number of unknown functions $f_{j}$ equals the number of variables $x_{i}$, i.e., $k=n$ but in general this assumption is irrelevant.

In this view, we will prove an analogue of Lemma 3.8 for a more general PDE system (16) (from which Lemma 3.8 can be easily derived by using the above substitution $\left.f_{i}=U_{x_{i}}\right)$. Recall that all the functions involved are assumed to be real-analytic.

Lemma 4.1 The following properties of (16) are equivalent:

- For any initial straight line $x_{1}=a_{1}, \ldots, x_{n}=a_{n}$ and any real-analytic initial conditions on it

$$
\begin{aligned}
f_{1}\left(a_{1}, \ldots, a_{n-1}, x_{n}\right) & =v_{1}\left(x_{n}\right), \\
f_{2}\left(a_{1}, \ldots, a_{n-1}, x_{n}\right) & =v_{2}\left(x_{n}\right), \\
\ldots & \\
f_{k}\left(a_{1}, \ldots, a_{n-1}, x_{n}\right) & =v_{k}\left(x_{n}\right),
\end{aligned}
$$

there locally exists a unique real-analytic solution $F=\left(\begin{array}{c}f_{1} \\ \vdots \\ f_{n}\end{array}\right)$ of (16).

- The following compatibility conditions are identically fulfilled in virtue of (16):

$$
D_{x_{i}} H_{j}=D_{x_{j}} H_{i}, \quad i, j=1, \ldots, n-1 \text {. }
$$

Proof We give a proof in the simplest non-trivial case, ${ }^{4}$ namely, for three variables $x, y, z$ and one function $f$ so that (16) becomes

$$
f_{x}=h_{1}\left(x, y, z, f, f_{z}\right) \text { and } f_{y}=h_{2}\left(x, y, z, f, f_{z}\right) .
$$

Assume that the condition $D_{x} h_{2}=D_{y} h_{1}$ holds identically in virtue of the system. In more detail,

$$
\begin{aligned}
D_{x} h_{2} & =\frac{\partial h_{2}}{\partial x}+\frac{\partial h_{2}}{\partial f} f_{x}+\frac{\partial h_{2}}{\partial f_{z}} f_{z x}=\frac{\partial h_{2}}{\partial x}+\frac{\partial h_{2}}{\partial f} h_{1}+\frac{\partial h_{2}}{\partial f_{z}} D_{z} h_{1}\left(x, y, z, f, f_{z}\right) \\
& =\frac{\partial h_{2}}{\partial x}+\frac{\partial h_{2}}{\partial f} h_{1}+\frac{\partial h_{2}}{\partial f_{z}}\left(\frac{\partial h_{1}}{\partial z}+\frac{\partial h_{1}}{\partial f} f_{z}+\frac{\partial h_{1}}{\partial f_{z}} f_{z z}\right) .
\end{aligned}
$$

\footnotetext{
4 The general case is not essentially different.
} 
And similarly

$$
D_{y} h_{1}=\frac{\partial h_{1}}{\partial y}+\frac{\partial h_{1}}{\partial f} h_{2}+\frac{\partial h_{1}}{\partial f_{z}}\left(\frac{\partial h_{2}}{\partial z}+\frac{\partial h_{2}}{\partial f} f_{z}+\frac{\partial h_{2}}{\partial f_{z}} f_{z z}\right)
$$

In these two expressions we see an additional formal variable $f_{z z}$. In this simple case the corresponding coefficients in both expressions take the form $\frac{\partial h_{1}}{\partial f_{z}} \frac{\partial h_{2}}{\partial f_{z}}$ and therefore coincide automatically. However in general it is not necessarily the case so that the equality of these coefficients should be considered as an extra condition additional to

$$
\frac{\partial h_{2}}{\partial x}+\frac{\partial h_{2}}{\partial f} h_{1}+\frac{\partial h_{2}}{\partial f_{z}}\left(\frac{\partial h_{1}}{\partial z}+\frac{\partial h_{1}}{\partial f} f_{z}\right)=\frac{\partial h_{1}}{\partial y}+\frac{\partial h_{1}}{\partial f} h_{2}+\frac{\partial h_{1}}{\partial f_{z}}\left(\frac{\partial h_{2}}{\partial z}+\frac{\partial h_{2}}{\partial f} f_{z}\right) .
$$

Identical fulfilment of this condition means that the functions in the right-hand side and the left-hand side coincide as functions of five independent variables $x, y, z, f$ and $f_{z}$.

Without loss of generality, we will assume that the parameters $a_{1}$ and $a_{2}$ in the initial condition vanish. For a given initial condition $f(0,0, z)=v(z)$ we construct the desired solution in two steps. We first construct a unique function of two variables $u(y, z)$ that is a local real-analytic solution of the Cauchy problem

$$
u_{y}=h_{2}\left(0, y, z, u, u_{z}\right), \quad u(0, z)=v(z) .
$$

The existence of $u(y, z)$ follows from the classical Cauchy-Kovalevskaya theorem. If the desired solution $f$ of (17) exists then necessarily $f(x, 0, z)=u(x, z)$.

The next step is solving the Cauchy problem

$$
f_{x}=h_{1}\left(x, y, z, f, f_{z}\right), \quad f(0, y, z)=u(y, z) .
$$

Again, a unique local real-analytic solution $f(x, y, z)$ exists by the Cauchy-Kovalevskaya theorem.

Thus we have constructed a function that satisfies the initial condition and solves the first equation of (17), as for the the second equation, it is fulfilled only on the plane $x=0$. Our goal is to show that $D_{x} h_{2}=D_{y} h_{2}$ guarantees that the second equation holds at all the points.

Lemma 4.2 Let $f$ satisfy the conditions

$$
f_{x}(x, y, z)=h_{1}\left(x, y, z, f, f_{z}\right) \text { and } f_{y}(0, y, z)=h_{2}\left(0, y, z, f, f_{z}\right) .
$$

Then if the compatibility condition $D_{x} h_{2}=D_{y} h_{1}$ is identically fulfilled, then

$$
f_{y}(x, y, z)=h_{2}\left(x, y, z, f, f_{z}\right) \text { for all } x, y, z \text {. }
$$


Proof Consider the functions

$$
\begin{aligned}
& \widehat{h}_{2}(x, y, z)=h_{2}\left(x, y, z, f(x, y, z), f_{z}(x, y, z)\right), \\
& \widehat{h}_{1}(x, y, z)=h_{1}\left(x, y, z, f(x, y, z), f_{z}(x, y, z)\right),
\end{aligned}
$$

and compute the partial derivative of $f_{y}-\widehat{h}_{2}$ w.r.t. $x$ :

$$
\begin{aligned}
\frac{\partial}{\partial x}\left(f_{y}-\widehat{h}_{2}\right) & =\frac{\partial}{\partial y} f_{x}-\frac{\partial h_{2}}{\partial x}-\frac{\partial h_{2}}{\partial f} f_{x}-\frac{\partial h_{2}}{\partial f_{z}} f_{z x} \\
& =\frac{\partial \widehat{h}_{1}}{\partial y}-\frac{\partial h_{2}}{\partial x}-\frac{\partial h_{2}}{\partial f} \widehat{h}_{1}-\frac{\partial h_{2}}{\partial f_{z}} \frac{\partial \widehat{h}_{1}}{\partial z} \\
& =\frac{\partial h_{1}}{\partial y}+\frac{\partial h_{1}}{\partial f} f_{y}+\frac{\partial h_{1}}{\partial f_{z}}\left(f_{y}\right)_{z}-\frac{\partial h_{2}}{\partial x}-\frac{\partial h_{2}}{\partial f} \widehat{h}_{1}-\frac{\partial h_{2}}{\partial f_{z}} \frac{\partial \widehat{h}_{1}}{\partial z}
\end{aligned}
$$

and taking into account the compatibility condition

$$
\frac{\partial h_{1}}{\partial f} f_{y}+\frac{\partial h_{1}}{\partial f_{z}}\left(f_{y}\right)_{z}-\frac{\partial h_{1}}{\partial f} \widehat{h}_{2}-\frac{\partial h_{1}}{\partial f_{z}} \frac{\partial \widehat{h}_{2}}{\partial z}=\frac{\partial h_{1}}{\partial f}\left(f_{y}-\widehat{h}_{2}\right)+\frac{\partial h_{1}}{\partial f_{z}}\left(f_{y}-\widehat{h}_{2}\right)_{z} .
$$

We see that the function $f_{y}-\widehat{h}_{2}$ satisfies the linear PDE

$$
\frac{\partial}{\partial x}\left(f_{y}-\widehat{h}_{2}\right)=\frac{\partial h_{1}}{\partial f}\left(f_{y}-\widehat{h}_{2}\right)+\frac{\partial h_{1}}{\partial f_{z}}\left(f_{y}-\widehat{h}_{2}\right)_{z} .
$$

Moreover for $x=0$ we have $f_{y}(0, y, z)-\widehat{h}_{2}(0, y, z)=0$. This implies that $f_{y}-\widehat{h}_{2}$ vanishes identically, that is,

$$
f_{y}(x, y, z)=h_{2}\left(x, y, z, f(x, y, z), f_{z}(x, y, z)\right),
$$

as stated.

The proof of the converse statement is obvious. Indeed, if $f$ is the solution of (17) with the initial condition $f\left(a_{1}, a_{2}, z\right)=u(z)$, then $f_{x y}=f_{y x}$ implies that $D_{y} H_{1}\left(x, y, z, f, f_{z}\right)=D_{x} H_{2}\left(x, y, z, f, f_{z}\right)$. Moreover, since $u(z)$ and $u_{z}(z)$ can be chosen arbitrarily for fixed $x=a_{1}, y=a_{2}$ and $z$, we see that $D_{y} H_{1}=D_{x} H_{2}$ holds identically (with $x, y, z, f$ and $f_{z}$ being formal independent variables), as required.

\section{Appendix B: Turiel extension construction and an alternative form of the main theorem}

Formulas (1), (2) from Theorem 2.1 can be naturally interpreted in terms of the Turiel extension construction [21]. This gives an additional proof that they indeed define a Nijenhuis operator that is compatible with the canonical symplectic structure $\sum_{i} \mathrm{~d} x_{i} \wedge \mathrm{d} p_{i}$. 
Recall that for an arbitrary Nijenhuis operator $A$ on a manifold $Q$, the Turiel construction provides a natural extension of $A$ to the cotangent bundle $M^{2 n}=T^{*} Q^{n}$ as follows. Consider local coordinates $x_{1}, \ldots, x_{n}$ on $Q$ and denote by $x_{1}, \ldots, x_{n}$, $p_{1}, \ldots, p_{n}$ the corresponding canonical coordinates on $T^{*} Q$. Next, consider the operator on $T^{*} Q$ given in these coordinates by

$$
L=\left(\begin{array}{cc}
A & 0 \\
S & A^{\top}
\end{array}\right),
$$

where

$$
S_{i j}:=\sum_{\alpha=1}^{n} p_{\alpha}\left(\frac{\partial A_{i}^{\alpha}}{\partial x_{j}}-\frac{\partial A_{j}^{\alpha}}{\partial x_{i}}\right) .
$$

By [21], this operator is Nijenhuis and compatible with the canonical symplectic structure $\sum_{i} \mathrm{~d} p_{i} \wedge \mathrm{d} x_{i}$ on $T^{*} Q$.

Example 4.3 By direct calculations we see that the "normal form" from Theorem 2.1 is in fact the Turiel extension of the Nijenhuis operator in the (first) companion form (7) with $\sigma_{i}=x_{i}$ : indeed, $S$ and $A$ from (2) are related by (19).

Observe that the Turiel construction is evidently geometric and does not depend on the choice of a coordinate system $x_{1}, \ldots, x_{n}$ in which we apply it. Indeed, $L$ is the recursion operators for two 2-forms $\omega$ and $\widetilde{\omega}$ each of which is geometric (see [11]): the first of them is canonical $\omega=-\mathrm{d} \theta, \theta=\sum p_{\alpha} \mathrm{d} x_{\alpha}$, the second is defined by the following invariant formula $\widetilde{\omega}=-\mathrm{d} \widetilde{\theta}$ with $\widetilde{\theta}=A^{*} \theta=\sum A_{j}^{i}(x) p_{i} \mathrm{~d} x_{j}$. One therefore may look for coordinate systems on $Q$ such that in the corresponding coordinate system on $T^{*} Q$ the extended operator $L$ takes a more convenient form. The interpretation of "convenient" may depend on the problem we are solving and on the situation in which the recursion Nijenhuis operator appeared. As an illustration, let us show that one can choose a coordinate system on $Q$ in such a way that $S$ in (18) vanishes.

We say that a Nijenhuis operator $A$ is in the second companion form if its matrix, in local coordinates $y_{1}, \ldots, y_{n}$, has the form (cf. (7))

$$
A=\left(\begin{array}{ccccc}
0 & 1 & 0 & \ldots & 0 \\
0 & 0 & 1 & \ldots & 0 \\
\vdots & \vdots & \ddots & \ddots & \vdots \\
0 & 0 & \ldots & 0 & 1 \\
-\sigma_{n}-\sigma_{n-1} & \ldots & \ldots & -\sigma_{1}
\end{array}\right)
$$

Not every operator of the form (20) is Nijenhuis. The next lemma gives necessary and sufficient condition for this property to hold.

Lemma 4.4 An operator A given by (20) is Nijenhuis if and only if

$$
\begin{aligned}
\mathrm{d}\left(A^{*} \mathrm{~d} y_{n}\right) & =0, \\
\mathrm{~d}\left(A^{* 2} \mathrm{~d} y_{n}\right) & =0 .
\end{aligned}
$$


Condition (21) simply means that the differential 1-form given by the last row of $A$ is closed, i.e., $\frac{\partial \sigma_{n-i+1}}{\partial y_{j}}=\frac{\partial \sigma_{n-j+1}}{\partial y_{i}}$ for any $i, j$; it is a linear condition on $\sigma_{i}$. The other condition (22) is nonlinear.

Proof We first recall (see e.g. [2, Definition 2.5]) that for an operator $A$, its Nijenhuis tensor $\mathcal{N}_{A}$ viewed as a mapping from 1 -forms to 2 -forms is given by

$$
\begin{aligned}
\mathcal{N}_{A}: \alpha \mapsto \beta(\cdot, \cdot):=\mathrm{d}\left(A^{* 2} \alpha\right)(\cdot, \cdot) & +\mathrm{d} \alpha(A \cdot, A \cdot) \\
& -\mathrm{d}\left(A^{*} \alpha\right)(A \cdot, \cdot)-\mathrm{d}\left(A^{*} \alpha\right)(\cdot, A \cdot) .
\end{aligned}
$$

If $A$ is Nijenhuis and both $\alpha$ and $A^{*} \alpha$ are closed, this formula implies that also $A^{* 2} \alpha$ is closed. For our $A$ given by (20), the forms $\mathrm{d} y_{1}$ and $\mathrm{d} y_{2}=A^{*} \mathrm{~d} y_{1}$ are closed. Thus, if $A$ is Nijenhuis, the form $\left(A^{*}\right)^{n} \mathrm{~d} y_{1}=A^{*} \mathrm{~d} y_{n}$ is closed as well, which gives us (21). Similarly, the form $\left(A^{*}\right)^{n+1} \mathrm{~d} y_{1}=A^{* 2} \mathrm{~d} y_{n}$ is closed, which gives us (22).

In order to prove Lemma 4.4 in the other direction, let us observe that since $\mathcal{N}_{A}$ is a tensor, it is sufficient to check that (23) vanishes for the basis forms $\alpha=\mathrm{d} y_{i}$, $i=1, \ldots, n$. For them each term in (23) is zero.

Example 4.5 The Turiel extension of the Nijenhuis operator $A$ in the second companion form is

$$
L=\left(\begin{array}{cc}
A & 0_{n} \\
0_{n} & A^{\top}
\end{array}\right),
$$

where $0_{n}$ is the zero $n \times n$ matrix. Indeed, for fixed $i$ the 1 -form $A_{i}^{\alpha} \mathrm{d} x_{\alpha}$ is just $A^{*(i-1)} \mathrm{d} x_{1}$; so the corresponding sum in (19) vanishes for $i<n$ trivially and for $i=n$ by (21).

We use this observation to give another canonical form for Poisson-Nijenhuis structures from Theorem 2.1. To this end we use a coordinate transformation $x \mapsto y$ that reduces the Nijenhuis operator $A$ from Theorem 2.1 (being in the first companion form)

$$
A=\left(\begin{array}{ccccc}
-x_{1} & 1 & 0 & \cdots & 0 \\
-x_{2} & 0 & 1 & \ddots & \vdots \\
\vdots & \vdots & \ddots & \ddots & 0 \\
-x_{n-1} & \vdots & & \ddots & 1 \\
-x_{n} & 0 & \cdots & \cdots & 0
\end{array}\right)
$$

to the second companion form (20). Suitable new coordinates $\left(y_{1}, \ldots, y_{n}\right)$ are easy to find by using [2, Proposition 2.1] which states that for any Nijenhuis operator $A$ and any $k \in \mathbb{N}$ we have

$$
\frac{1}{k} \mathrm{~d} \operatorname{tr} A^{k}=\frac{1}{k-1} A^{*} \mathrm{~d} \operatorname{tr} A^{k-1} .
$$


Let us set

$$
y_{1}=-\operatorname{tr} A, \quad y_{2}=-\frac{1}{2} \operatorname{tr} A^{2}, \quad \ldots, \quad y_{n}=-\frac{1}{n} \operatorname{tr} A^{n} .
$$

Notice that the transition formulas $x \mapsto y$ express the traces $y_{1}, \ldots, y_{n}$ of powers of $A$ (with some coefficients) in terms of the coefficients of its characteristic polynomial $x_{1}, \ldots, x_{n}$. Such a transformation is known to be invertible and polynomial in both directions (see below the formulas for the inverse transformation $y \mapsto x$ ).

From (24) we see that $A^{*} \mathrm{~d} y_{i}=\mathrm{d} y_{i+1}, i \in 1, \ldots, n-1$, meaning that the first $n-1$ rows of $A$ in the $y$-coordinate system are as needed, i.e., in these coordinates $A$ takes the second companion form (20). The corresponding functions $\sigma_{k}(y)$ in the last row of (20) can be explicitly constructed. Indeed, $\sigma_{k}$ are the coefficients of the characteristic polynomial $\chi_{A}(t)=\operatorname{det}(t \cdot \operatorname{Id}-A)=t^{n}+\sigma_{1} t^{n-1}+\cdots+\sigma_{n}$ so that the functions $\sigma_{k}\left(y_{1}, \ldots, y_{n}\right)$ coincide with $x_{k}$ and are given by the condition

$$
\sigma_{k}\left(-\operatorname{tr} A,-\frac{1}{2} \operatorname{tr} A^{2}, \ldots,-\frac{1}{n} \operatorname{tr} A^{n}\right)=\text { coefficient of } t^{n-k} \text { in } \chi_{A}(t)
$$

This condition is well known and the functions $\sigma_{i}$ satisfying (25) can be obtained from the famous Newton-Girard polynomials (by appropriate rescaling and changing sings). They depend neither on the matrix $A$ nor on the dimension and are given by the following universal formula, see e.g. [10]:

$$
\sigma_{k}=\sum_{\substack{m_{1}+2 m_{2}+\cdots+k m_{k}=k \\ m_{1} \geqslant 0, \ldots, m_{k} \geqslant 0}} \prod_{\substack{k \\ i=1}}^{y_{i}^{m_{i}}}
$$

For example, the first five functions $\sigma_{1}, \ldots, \sigma_{5}$ are given by

$$
\begin{aligned}
& \sigma_{1}=y_{1} \\
& \sigma_{2}=y_{2}+\frac{1}{2} y_{1}^{2} \\
& \sigma_{3}=y_{3}+y_{1} y_{2}+\frac{1}{6} y_{1}^{3} \\
& \sigma_{4}=y_{4}+\frac{1}{24} y_{1}^{4}+\frac{1}{2} y_{2} y_{1}^{2}+y_{1} y_{3}+\frac{1}{2} y_{2}^{2}, \\
& \sigma_{5}=y_{5}+\frac{1}{120} y_{1}^{5}+\frac{1}{6} y_{2} y_{1}^{3}+\frac{1}{2} y_{3} y_{1}^{2}+\frac{1}{2} y_{1} y_{2}^{2}+y_{1} y_{4}+y_{3} y_{2} .
\end{aligned}
$$

Now, if we naturally extend the coordinate transformation $x \mapsto y$ from $Q$ to the cotangent bundle $M=T^{*} Q$, then in new coordinates $\left(y, p_{y}\right)$ the operator $L$ will be the Turiel extension of $A$ in the second companion form (20), whereas $\omega$ will remain canonical. Taking into account Example 4.5, we get the following alternative version of Theorem 2.1. 
Theorem 4.6 Let $L$ and $\omega$ be compatible (i.e., define a Poisson-Nijenhuis structure on $\left.M^{2 n}\right)$ and real-analytic. Suppose that at a point $p \in M^{2 n}$ the differentials $\mathrm{d} \operatorname{tr} L, \ldots, \mathrm{d} \operatorname{tr} L^{n}$ are linearly independent. Then there exists a local coordinate system $y_{1}, \ldots, y_{n}, p_{1}, \ldots, p_{n}$ such that $\omega=\sum_{i} \mathrm{~d} y_{i} \wedge \mathrm{d} p_{i}$ and $L$ is given by the matrix

$$
L=\left(\begin{array}{cc}
A & 0_{n} \\
0_{n} & A^{\top}
\end{array}\right),
$$

where A is given by (20) with $\sigma_{k}$ by (26).

Acknowledgements The authors are grateful to the referee for valuable remarks and suggestions.

Open Access This article is licensed under a Creative Commons Attribution 4.0 International License, which permits use, sharing, adaptation, distribution and reproduction in any medium or format, as long as you give appropriate credit to the original author(s) and the source, provide a link to the Creative Commons licence, and indicate if changes were made. The images or other third party material in this article are included in the article's Creative Commons licence, unless indicated otherwise in a credit line to the material. If material is not included in the article's Creative Commons licence and your intended use is not permitted by statutory regulation or exceeds the permitted use, you will need to obtain permission directly from the copyright holder. To view a copy of this licence, visit http://creativecommons.org/licenses/by/4.0/.

\section{References}

1. Bolsinov, A., Matveev, V.S., Miranda, E., Tabachnikov, S.: Open problems, questions, and challenges in finite-dimensional integrable systems. Philos. Trans. R. Soc. A 376(2131), \# 20170430 (2018). arXiv: 1804.03737

2. Bolsinov, A.V., Konyaev, A.Yu., Matveev, V.S.: Nijenhuis Geometry (2019). arXiv:1903.04603

3. Bolsinov, A., Konyaev, A., Matveev, V.: Nijenhuis Geometry III: gl-Regular Nienhuis Operators (2020). arXiv:2007.09506

4. Ferapontov, E.V.: Compatible Poisson brackets of hydrodynamic type. J. Phys. A 34(11), 2377-2388 (2001)

5. Ferapontov, E.V., Lorenzoni, P., Savoldi, A.: Hamiltonian operators of Dubrovin-Novikov type in 2D. Lett. Math. Phys. 105(3), 341-377 (2015)

6. Frazer, R.A., Duncan, W.J., Collar, A.R.: Elementary Matrices and Some Applications to Dynamics and Differential Equations. Cambridge University Press, New York (1960)

7. Gelfand, I.M., Zakharevich, I.: On the local geometry of a bi-Hamiltonian structure. In: The Gel'fand Mathematical Seminars, 1990-1992, pp. 51-112. Birkhäuser, Boston (1993)

8. Goldschmidt, H.: Integrability criteria for systems of nonlinear partial differential equations. J. Differential Geom. 1(3-4), 269-307 (1967)

9. Haantjes, J.: On $X_{m}$-forming sets of eigenvectors. Indag. Math. 17, 158-162 (1955)

10. https://en.wikipedia.org/wiki/Newton's_identities

11. Ibort, A., Magri, F., Marmo, G.: Bihamiltonian structures and Stäckel separability. J. Geom. Phys. 33(3-4), 210-228 (2000)

12. Konyaev, A.Yu.: Nijenhuis geometry II: Left-symmetric algebras and linearization problem. Diff. Geom. Appl. 74, \# 101706 (2021). arXiv:1903.06411

13. Kosmann-Schwarzbach, Y., Magri, F.: Poisson-Nijenhuis structures. Ann. Inst. H. Poincaré Phys. Théor. 53(1), 35-81 (1990)

14. Kostant, B.: Lie group representations on polynomial rings. Amer. J. Math. 85, 327-404 (1963)

15. Lorenzoni, P., Magri, F.: A cohomological construction of integrable hierarchies of hydrodynamic type. Int. Math. Res. Not. 2005(34), 2087-2100 (2005)

16. Magri, F.: A simple model of the integrable Hamiltonian equation. J. Math. Phys. 19(5), 1156-1162 (1978) 
17. Magri, F., Morosi, C.: A geometrical characterization of integrable Hamiltonian systems through the theory of Poisson-Nijenhuis manifolds. Preprint (Università di Milano, Dipartimento di Matematica 'F. Enriques', Quaderno S 19/1984)

18. Magri, F.: Haantjes manifolds with symmetry. Theoret. and Math. Phys. 196(2), 1217-1229 (2018)

19. Nijenhuis, A.: $X_{n-1}$-forming sets of eigenvectors. Proc. Kon. Ned. Akad. Amsterdam 54, 200-212 (1951)

20. Turiel, F.-J.: Classification locale simultanée de deux formes symplectiques compatibles. Manuscripta Math. 82(3-4), 349-362 (1994)

21. Turiel, F.J.: Classification of $(1,1)$ tensor fields and bihamiltonian structures. In: Singularities and Differential Equations. Banach Center Publications, vol. 33, pp. 449-458. PAN, Warszawa (1996). http://matwbn.icm.edu.pl/ksiazki/bcp/bcp33/bcp33140.pdf

Publisher's Note Springer Nature remains neutral with regard to jurisdictional claims in published maps and institutional affiliations. 